\title{
Diversidad de Ericáceas con frutos comestibles, potencialidades para su manejo y estado de conservación en la región del Cusco, Perú
}

\section{Diversity of Ericaceae with edible fruits, potentialities for their management and conservation status in Cusco region, Peru}

\section{Isau Huamantupa-Chuquimaco ${ }^{1}$, Rosa Urrunaga Soria ${ }^{2}$ \& Alfredo Tupayachi Herrera ${ }^{3}$}

\author{
${ }^{1}$ Centro Ecológico INKAMAZONIA, Valle de Kosñipata, via Cusco-RB Manú. Cusco, Perú. e- \\ mail: andeanwayna@gmail.com \\ ${ }^{2}$ Centro de Estudios de Plantas Alimenticias y Medicinales (CEPLAM). Universidad Nacional \\ de San Antonio Abad del Cusco (UNSAAC). Av. de la Cultura 733 Cusco, Perú. \\ ${ }^{3}$ Sociedad Botánica del Cusco (SBC). Av. de la Cultura 733 Cusco, Perú.
}

\section{Resumen}

La importancia de conocer especies silvestres de frutas consumidas por las comunidades locales y sus potencialidades para su aprovechamiento, son de los principales beneficios provenientes de la biodiversidad, para suplir la demanda de alimentos por la población humana. En el presente estudio se alcanza los resultados de investigaciones de más de 20 años, referente a frutales comestibles de la familia Ericaceae en la región del Cusco; para este fin se propuso como objetivos: identificar la diversidad de especies de Ericáceas con frutos comestibles consumidas por los pobladores locales, analizar las potencialidades de manejo para su aprovechamiento y determinar el estado de conservación a través de las categorías de amenaza. Se registraron 51 especies de Ericáceas con frutos consumidos, distribuidos en 14 géneros, los más diversos fueron Demosthenesia con 10 especies, Disterigma 8, Cavendishia y Thibaudia con 6, de estas 12 son las especies que gozan de mayor frecuencia de consumo por parte de las poblaciones locales; en cuanto a las potencialidades se identificaron y analizaron 10 especies con alto potencial para ser manejados en cultivos locales, de ellas actualmente son expendidas en algunos mercados rurales: Vaccinium floribundum y esporádicamente Thibaudia crenulata y Pernettya prostrata; en lo referente al estado de conservación se determinaron 13 especies con categorías de amenaza, de ellas 10 están consideradas en peligro crítico (CR), siendo Demosthenesia el género con más especies categorizadas. Estos resultados evidencian la existencia de una enorme riqueza de especies de Ericaceae con los frutos comestibles en la región del Cusco. Esta riqueza como recurso intangible, cuenta con potenciales sobresalientes, representados por especies con aptitudes agronómicas, nutraceúticas, medicinales, ornamentales, para restauración ecológica, entre otros. Por tales razones, consideramos que deben ser estudiados con énfasis para un aprovechamiento adecuado y puedan formar parte de alternativas de desarrollo futuro, que a su vez mejoren la calidad de vida, especialmente de las poblaciones locales.

Palabras Clave. Antioxidante, Frutales silvestres, Inca, Mulluntuy, Tumana.

\begin{abstract}
The importance of knowing wild species of fruits consumed by local communities and their potential for use, is some of the main benefits from biodiversity, to meet the demand for food by the human population. In the present study the results of investigations of more than 20 years are documented, referring to edible fruit species of the Ericaceae family in the Cusco region; to this aim, the following objectives were proposed: identify the diversity of Ericaceae species with edible fruits consumed by local populations, analyze the management potential for their use and to determine the state of conservation through the threat categories. 51 species of Ericaceae with consumed fruits were recorded, distributed in 14 genera, the most diverse were Demosthenesia with 10 species, Disterigma 8, Cavendishia and Thibaudia with 6 , of these 12 are the species that enjoy the highest frequency of consumption by the local populations; Regarding the potential, 10 species with high potential to be managed in local crops were identified, of
\end{abstract}


currently sold in some local markets: Vaccinium floribundum and sporadically Thibaudia crenulata and Pernettya prostrata; Regarding the conservation status, 13 species were registered with threatened categories, of which 10 are considered critically endangered (CR), with Demosthenesia being the genus with the most categorized species. These results show the existence of an enormous richness of Ericaceae species with edible fruits, in the Cusco region. This richness as an intangible resource, has outstanding potentials, represented by species with agronomic, nutraceutical and medicinal aptitudes beneficial to health, such as ornamentals, for ecological restoration, among others. For these reasons, we consider that they should be studied with emphasis for an adequate use and can be part of future development alternatives, which in turn improve the quality of life, especially of local populations.

Palabras Clave. Antioxidant, Inca, Mulluntuy, Tumana, Wild fruit.

\section{Introducción}

La familia Ericaceae en los bosques neotropicales es una de las más representativas, principalmente habitan los bosques montañosos, actualmente esta representada por aproximadamente 46 géneros y 900 especies, con cerca del 94\% de especies endémicas (Luteyn, 2002, 2004; Pedraza, 2010). Los géneros más ricos en especies son Cavendishia con 130 especies, Psammisia con 70, Thibaudia con 60, Gaylussacia con 42, Vaccinium con 40, Macleania con 40, Disterigma con 40, Gaultheria con 37 y Ceratostema con 35 (Smith, 2004). Siendo uno de los principales centros de diversificación las montañas de la región andina (Kron et al., 2002). Para el Perú, se reportan aproximadamente 145 especies distribuidas en 22 géneros (Brako \& Zarucchi, 1993; Huamantupa, 2009; Luteyn \& PedrazaPeñalosa, 2008; Pedraza et al., 2015). Los miembros de esta familia presentan hábitos variados, desde arbustos epífitos o hemiepífitos hasta subarbustos epífitos y terrestres. La mayoría de las epífitas y hemiepífitas presentan un lignotubérculo que es el crecimiento prolongado del hipocótilo (Luteyn \& PedrazaPeñalosa, 2008).

En cuanto a los usos las Ericaceae, principalmente son utilizadas para la ornamentación y de algunas especies son consumidas los frutos, pero muchas de estas aún son consideradas como especies subutilizadas dándole poco valor e importancia (Padulosi et al., 2004; Smith, 2004). También, las Ericáceas juegan un importante rol ecológico por ser fuente de alimento (néctar, frutos y corolas) para especies de aves como colibríes, passeridos, mamíferos menores y mayores como el oso de anteojos (Tremarctos ornatos) y otros; desde el punto de vista socioeconómico en las provincias cusqueñas son utilizadas como medicinales y alimenticias con el consumo de los frutos y corolas crasas por parte de las comunidades locales (Huamantupa, 2010; Moraes et al., 2006).

Hoy en día el consumo de frutos se ha incrementado por la demanda alimentaria de la población humana, buscando nuevas alternativas para su aprovechamiento, en este sentido las Ericaceae son consideradas como potenciales para la alimentación y como medicinales ya que han demostrado tener atributos nutraceúticos, como es el caso de la tumana (Vaccinium floribundum) y (Thibaudia crenulata) en los andes del neotrópico; la primera destaca por presentar azúcares, fibra, lípidos, minerales y vitaminas, proantocianidinas, antocianidinas, flavonoides, que se utilizan como colorantes alimentarios, las que muchas veces también actúan sobre afecciones y enfermedades como el reumatismo, fiebres, cólicos y hasta son 
recomendadas contra la diabetes (Morales, 2011; CESA, 1993; Abreu et al., 2008; Mostacero et al., 2017). Por todos estos atributos las frutas son los alimentos de mayor consumo a nivel mundial, especialmente en zona andina de Latinoamérica, como es el caso de la familia Ericaceae (Sanjinés et al., 2006).

Para el sur peruano y la región del Cusco previamente se ha documentado la existencia de algunas especies de Ericaceae que son aprovechadas como frutales de consumo directo como: Pernettya prostrata y Vaccinium floribundum (conocidos localmente como tumana y mulluntu), las cuales son asociados al conocimiento ecológico tradicional, con experiencias de manejo desde los tiempos preincas e inca, (Vargas, 1994; Huamantupa, 2010). En este contexto el presente documento alcanza una minuciosa diagnosis de las especies consumidas como frutales en la región del Cusco. Para ello se propuso como objetivos, a) identificar las especies de Ericaceae con frutos que se consumen en la región del Cusco, b) analizar sus potencialidades de manejo y uso que se le da en la región Cusco y c) determinar su estado de conservación a través de las categorías de amenaza en la región del Cusco.

\section{Material y métodos}

\section{Área de Estudio}

El área de estudio corresponde a toda la región de Cusco, dentro de ellas los ámbitos de las provincias de Anta, Calca, La Convención, Paucartambo, Quispicanchis y Urubamba $\left(13^{\circ} 01^{\prime}, 72^{\circ} 02^{\prime}\right.$; $11^{\circ} 43^{\prime}, 73^{\circ} 28^{\prime} ; 12^{\circ} 46^{\prime}$, 73⒊'; $\left.12^{\circ} 17^{\prime}, 71^{\circ} 59^{\prime}\right)$, todas ellas con sus diferentes distritos. Estas localidades comprenden hábitats diversos que integran a los pajonales húmedos, bosques esclerófilos montanos, bosques mixtos transicionales de pajonales y arboles enanos, bosque húmedo montano, valles interandinos, bosques pre montanos, y los bosques amazónicos, en éstas los regímenes de precipitación son variables con regímenes de 2500 hasta $4500 \mathrm{~mm}$ (Aragon \& Chuspe, 2018; Huamantupa, 2010), estás están inmersas entre los limites altitudinales de 340 msnm por el bajo Urubamba hasta los altos nevados y pajonales que llegan a los 4800 msnm hasta donde registran especies de Ericaceae.

\section{Metodología}

El estudio comprende procedimientos que implicaron la toma de datos en campo, entrevista a pobladores locales, que incluyeron la colecta de especímenes, la revisión de bases de datos de herbarios la identificación de estas, el análisis de sus potencialidades de aprovechamiento y la determinación de las categorías de amenazas las cuales se detallan a continuación.

\section{Diversidad de Ericáceas con frutos comestibles}

Para identificar las especies de Ericaceae con frutos consumidos por las poblaciones locales, se comenzó a recopilar la información desde el año 2001 hasta el presente. Para ello nos valimos de entrevistas estructuradas y observaciones in situ con diversos pobladores en los diferentes distritos de las provincias descritas en el área de estudio. También, información etnobotánica fue obtenida y recogidas de las etiquetas y bases de datos de los especímenes de diferentes herbarios consultados: CUZ, F, HOXA, MO, NY, USM y US (acrónimos de acuerdo a Thiers et al., 2019).

Para diferenciar la frecuencia de consumo se asignó tres categorías, siendo a) preferencia menor (1), aquella que ocasionalmente es consumida por las personas, b) preferencia 
regular (2), aquella especie que tiene cierta demanda por su sabor dulce, tamaño considerable del fruto y c) preferencia alta (3), porque son consumidas de manera bastante frecuente por el sabor agradable por su tamaño grande $(\geq 2.5 \mathrm{~cm}$ circunferencia) $\mathrm{y} / \mathrm{o}$ por la cantidad de pulpa (mesocarpio). Se consideraron otras variables importantes como el nombre vernacular, hábito de crecimiento, gradiente altitudinal, provincia donde habita y el tipo de bosque donde se desarrollan.

Las colecciones comprenden todas aquellas que se realizaron desde los primeros botánicos e investigadores de la flora cusqueña y peruana, de los que destacan las valiosas colecciones de F. L. Herrera, A. Weberbauer y C. Vargas quienes dieron comienzo a la formación del herbario CUZ, y luego los aportes de los proyectos diversidad florística del Santuario Histórico de Machupicchu y el proyecto Flora del Perú en las cuencas del Vilcanota y adyacentes a cargo del Jardín Botánico de Missouri y de la Universidad Nacional de San Antonio Abad del Cusco (UNSAAC). Gran parte de los especímenes colectados se encuentran en el herbario CUZ de la UNSAAC y otros nacionales como el HOXA, USM, HUT y MOL, también en herbarios internacionales de MO, F, US y el NY con el cual se desarrollaron proyectos enfocados en la sistemática de familia Ericaceae como el Neotropical blueberries.

Las determinaciones e identificaciones taxonómicas han sido progresivas con el tratamiento de los diversos géneros por diversos autores, donde estacan los tratamientos de los géneros Demosthenesia, Orthaea, Psammisia, Cavendishia y Siphonandra y en menor medida las otras en las cuales aún se tienen vacíos, también coadyuvados por la gran diversidad de taxones (Taxa) en los bosques andino amazónicos de la región Cusco. Siempre que fue posible, se revisaron los tipos de las descripciones prínceps (primer espécimen) y/o fotótipos y, en todos los casos, las descripciones originales a nuestro alcance taxonómico, también se consultó con la literatura especializada que comprenden a (Flora Neotrópica: Luteyn, 1983; 1995), catálogo de las Gymnospermas y Angiospermas del Perú (Brako \& Zarucchi, 1993) y documentos de especies nuevas y tratamientos ecológicos desarrollados por (Huamantupa, 2008, 2010; Luteyn \& Ortiz, 2008; Pedraza-Peñalosa, 2004, 2010).

\section{Potencialidades para el uso y manejo de Ericáceas con frutos comestibles}

Para identificar y analizar las potencialidades de los frutales se ha considerado el enfoque definido por GonzalesVigil, (2003), quién considera que existen potencialidades tangibles e intangibles, en mayor o menor medida, en un determinado lugar y estás como en los taxones de plantas presentan un alto potencial de aprovechamiento, pero que no están siendo utilizados plenamente -o no se les está utilizando- adecuadamente en el desarrollo de la generación actual o futura, y que a su vez permita obtener beneficios que sean capaces de dinamizar las economías donde están localizadas y mejorar los niveles de desarrollo humano sostenible de las poblaciones naturales. Este estudio se enfocó principalmente en las especies que han tenido alguna experiencia de cultivo local y algún otro tipo de manejo de aprovechamiento asociado con el consumo de los frutos, tanto en áreas silvestres y zonas de cultivo de las comunidades. También, para este mismo fin se complementaron informaciones provenientes de fuentes bibliográficas de otras zonas. 


\section{Estado de conservación de Ericáceas con frutos comestibles}

Para determinar el estado de conservación nos hemos valido de los datos publicados y establecidos para las especies de Ericaceae en las obras de León (2006), León et al. (2007), DS. 064 del Ministerio de agricultura, en ellos se consideran los criterios establecidos por la UICN (2001). También, de acuerdo a nuestro estudio y análisis proponemos algunas categorías dentro de la región Cusco.

\section{Resultados}

\section{Diversidad de Ericáceas con frutos comestibles}

Se revisaron un total de 1630 colecciones de especímenes depositados en los diferentes herbarios: CUZ, HOXA, USM, HUT y MOL, $\mathrm{MO}$, F, US y NY, se logró entrevistar a 85 pobladores. Hemos identificado 51 especies con los frutos que se consumen, distribuidas en 14 géneros, los más diversos fueron los géneros Demosthenesia con 10 especies, Disterigma 8, Cavendishia y Thibaudia con 6, las otras diez estuvieron representadas con menos de 5 especies. De acuerdo con la distribución geográfica se registraron en seis provincias de la región Cusco, de ellas corresponden 41 especies para La Convención, 32 para Paucartambo, 32 para Calca, 30 para Urubamba, 9 en Quispicanchis y una en Anta (Tabla 1).

La distribución en cuanto al tipo de bosque y gradiente altitudinal es variable ya que se registra algunas especies Amazónicas como Psammisia amazonica y Satyria panurensis que son consumidas por etnias amazónicas, sin embargo el mayor rango está comprendida entre los 2000 a $3500 \mathrm{msnm}$ que comprende al bosque montano alto, bosque montano bajo, bosque de transición de pajonal y arbustal, seguida entre
1500 hasta los $2000 \mathrm{msnm}$, correspondiente al bosque montano bajo y bosques pre montanos amazónicos y luego las especies netamente altoandinas por encima de los $3500 \mathrm{msnm}$ en los pajonales húmedos y valles inter andinos. Las especies de mayor densidad de colección en las 6 Provincias fueron Cavendishia bracteata, Gaultheria glomerata Siphonandra elliptica y Vaccinium floribundum.

En cuanto a los hábitos de crecimiento más del $70 \%$ lo conforman especies con hábito de arbustos terrestres y hemiepífitos a la vez, es decir que ambos hábitos se pueden encontrar en el bosque de manera natural, solamente $C$. bracteata es la única considerada como árbol, pero que también puede ser hemiepífita.

Las especies de mayor frecuencia de consumo corresponde a 12: Demosthenesia mandonii, Macleania rupestris, Orthaea bullata, Orthaea carnosiflora, Orthaea ferreyrae, Pernettya prostrata, Psammisia amazonica, Siphonandra elliptica, Thibaudia crenulata, Thibaudia diphylla, Thibaudia dudleyi y Vaccinium floribundum, de regular frecuencia son 16 y de menor frecuencia corresponde a 23 especies (Tabla 1).

Potencialidades de manejo de Ericáceas con frutos comestibles

Tomando en cuenta el nuevo enfoque de potencialidades, consideramos a las especies registradas de Ericáceas en la región Cusco como del tipo intangible, las cuales están vinculados al conocimiento tradicional adquirido por las comunidades locales a través de generaciones que fueron transferidas a través de las vivencias del consumo de los frutos y relatos orales, este mismo conocimiento también va estrechamente relacionado al conocimiento del entorno abiótico como las temporadas de cosechas, suelos donde habitan, agentes dispersores y polinizadores, entre otros. 
Tabla 1. Especies de la familia Ericaceae consumidas como frutal en la región de Cusco. Tipo de Vegetación: BM (Bosque montano); BA (Bosque amazónico); Bosque Montano húmedo (BMH). Provincias: An (Anta), Ca (Calca), LC (La Convención), $\mathrm{Pa}$ (Paucartambo), Qu (Quispicanchis) y Ur (Urubamba).

\begin{tabular}{|c|c|c|c|c|c|c|c|}
\hline Especie & $\begin{array}{c}\begin{array}{c}\text { Frecuencia } \\
\text { consumo }\end{array} \\
\end{array}$ & Nombre vernacular & $\begin{array}{l}\text { Tipo } \\
\text { Bosque }\end{array}$ & Hábito & $\begin{array}{l}\text { Rango } \\
\text { altitud }\end{array}$ & Provincias & Veucher \\
\hline Gavendishic bracteata (Ruiz \& Pav. ex J. St.) Heereld & 1 & Pepino seera, Gapqlille & BM & $A, a H, a T$ & $1900-3700$ & $\mathrm{~Pa}_{\mathrm{a}} \mathrm{Ur}_{\mathrm{r}} \mathrm{LC}, \mathrm{Ca}, \mathrm{Qu}$ & I. Huamantupa 2314 (CUZ, MO) \\
\hline Cavendishie semplectens Hemsl. & 2 & Sixalillla & BMH & aH & $300-1200$ & Qu & I. Huamantura et al. $\mathrm{g}(\mathrm{CUZ})$ \\
\hline Cavendishia martii (Meisn.) A.C. Sm. & 2 & Huiluntu, Monte frutilla, Merowuntux & BM & $\mathrm{aH}, \mathrm{aT}$ & $1950-3700$ & $\mathrm{~Pa}_{2} \mathrm{Ur}, \mathrm{Ca}, \mathrm{LC}$ & I. Huamantupa 9414 (CUZ, MO, USM) \\
\hline Cavendishie nobilis Lindl. & 1 & Monte gapuli & BM & aH, aT & $2430-2800$ & $\mathrm{Ca}, \mathrm{LC}$ & B. Pexten 1435 (MO) \\
\hline Cavendishie pubercens (Kunth) Hemsl. & 1 & Capulille & BM & $\mathrm{aH}, \mathrm{aT}$ & $1500-2301$ & LC & G. Calatayud 4660 (CUZ, HUT, MO, USM) \\
\hline Carendishie targpotand (Meisn.) Benth. \& Hook. f. & 1 & Murmuntex & $\mathrm{BA}, \mathrm{BM}$ & $\mathrm{aH}, \mathrm{aT}$ & $800-1500$ & $\mathrm{~Pa}_{\mathrm{Q}} \mathrm{Qu}$ & I. Huamantura et al. gn (CUZ) \\
\hline Demesthenesia amicerum (Sleumer) Sleumer & 2 & Mulluntux & BM & aH, aT & $2000-3504$ & LC, Ur & L. Valenzuela 8105 (CUZ, MO, USM) \\
\hline Demesthenesia buxifolig (Fielding \& Gardner) A.C. Sm. & 2 & Mulluntux & $\mathrm{BM}, \mathrm{PA}$ & $\mathrm{aH}, \mathrm{aT}$ & $2656-3600$ & LC & L. Valenzuela 8014 (CUZ, MO, USM) \\
\hline Demerethenesia serdifolia Lutevn & 2 & Mulluntux & BM & $\mathrm{aH}, \mathrm{aT}$ & $2900-3400$ & LC & T. R. Dudley 11173 (NA, USM) \\
\hline Demersthereesia dudleri D.R. Simpson & 2 & Mullentux & BMH & $\mathrm{aH}, \mathrm{aT}$ & $2600-3300$ & LC & I. Huamantupa et al. (CUZ) \\
\hline Demersthenesia mandenix (Britton) A.C. Sm. & 3 & Mellluntux & BMH & $\mathrm{aH}, \mathrm{aT}$ & $2300-4150$ & $\mathrm{LC}, \mathrm{Pa}_{\mathrm{a}} \mathrm{Ur}, \mathrm{Ca}, \mathrm{Qu}$ & I. Huamantupa et al. 6855 (CUZ, MO) \\
\hline Demersthenecia mattriguenks Huamantupa. & 1 & & $\mathrm{BA}$ & $\mathrm{aH}$ & $1744-1800$ & LC & G. Calatayud et al. 3415 (CUZ, MO) \\
\hline Demerthenesia pearcer (Britton) A.C. Sm. & 1 & Mulluntux & $\mathrm{PA}$ & saH & $2100-3880$ & LC & I. Huananatupa et al 6954 (CuZ, MO) \\
\hline Remesthenesia sRectabilis (Busbu) A.C. Sm. & 2 & Mulluntux & BMH & $\mathrm{aH}, \mathrm{aT}$ & $2600-3500$ & $\mathrm{LC}, \mathrm{Ca}, \mathrm{Ux}, \mathrm{Pa}$ & I. Huamantupa 9472 (CUZ, MO, USM) \\
\hline Remesthenesia vilcabambensis Lutevn & 1 & Mulluntux & BM & $\mathrm{aH}, \mathrm{aT}$ & $2301-3000$ & $\mathrm{LC}, \mathrm{Ca}$ & G. Calatayud 4562 (CUZ, MO) \\
\hline Demersthenesia weberbqueri (Sleumer) Sleumer & 1 & Sulluntux & BM & saH & $3100-3500$ & LC & J. L. Lutexn 6465 (MO) \\
\hline Diegenesie boliviana (Britton) Sleumer & 1 & & BM & aH, aT & $2007-2301$ & $\mathrm{Ca}, \mathrm{LC}, \mathrm{Pa}_{2}, \mathrm{Ur}$ & E. Suclli 2801 (CUZ, HUT, MO, USM) \\
\hline Riegenesie vergasiane Sleumer & 1 & & BM & aH, aT & $900-3000$ & $\mathrm{Ca}, \mathrm{LC}, \mathrm{Pa}$ & V.H. Qquelltén 31 (CuZ, MO, USM, WFu) \\
\hline Disterigme scuminatum (Kunth) Nied. & 1 & Huilinte & $\mathrm{PA}$ & sath, sat & $3000-3500$ & $\mathrm{Hx}, \mathrm{Pa}_{2}$ & I. Huamantupa 3216 (CUZ) \\
\hline Disterigene glaterneider (Kunth) Nied. & 2 & & BM & sat & $2800-3500$ & $\mathrm{Ca}, \mathrm{LC}, \mathrm{Pa}$ & B. Peyton \& S.T. Peyton 1353 (MO) \\
\hline Disterigand empetrifolium (Kunth) Rrude & 2 & & BMH & $\operatorname{sath}$ & $2600-3600$ & $\mathrm{Ca}, \mathrm{LC}, \mathrm{Pa}$ & A. Tupayachi H. 37 (MO) \\
\hline Disterigme humbeldtii (Klotzsch) Nied. & 1 & & BMH & saH, saT & $2300-3000$ & Ux & I. Huamantupa et al. 3156 (CUZ, MO) \\
\hline Disterigma mickephrllum (G. Don) Lutevn & 1 & & BM & sath, sat & $2450-3100$ & $\mathrm{~Pa}$ & V.H. Qquellén 8 (HUT, MO, USM, WFU) \\
\hline Disterigme eratume (Busbrv) S.F. Blake & 1 & Yurachuilinte & BM & sat, sat & $1820-2800$ & $\mathrm{~Pa}_{9} \mathrm{Ux}$ & L. Valenzuela et al. 2844 (CUZ, MO) \\
\hline Disterique pernettroider (Griseb. ex Wedd.) Nied. & 2 & & BM & saT & $2500-3600$ & $\mathrm{Ca}, \mathrm{LC}, \mathrm{Pa}_{\mathrm{a}} \mathrm{Ux}, \mathrm{Qu}$ & I. Huanantura 9892 (CUZ) \\
\hline Risterigere ulei Sleumer & 2 & & BMH & $\mathrm{saH}, \mathrm{saT}$ & $1900-2600$ & $\mathrm{~Pa}$ & E. M. Ortiz V. 715 (MO, USM) \\
\hline Gqultterda buxifelig Willd. & 2 & Thumeana, Monte gapuli, Pycapichinche & BM & sath & $1900-2700$ & $\mathrm{Ca}, \mathrm{Ur}_{\mathrm{r}}, \mathrm{Pa}_{\mathrm{a}}$ & I. Huamantupa 4359 (CUZ, MO) \\
\hline Gqultherida erecta Vent. & 1 & Mullaca, Monte capuli & BM & sath & $2600-3200$ & $\mathrm{Ca}, \mathrm{LC}$ & I. Huamantupa 4362 (CUZ, MO) \\
\hline Gqultherie slomerats (Cax.) Sleumer & 1 & Lacea Lacca & BM & sat & $2300-4000$ & $\mathrm{Ca}, \mathrm{LC}, \mathrm{Pa}, \mathrm{Ur}$ & W. L. Galiano et al., 6489 (CUZ) \\
\hline Macleania rupestris (Kunth) A.C. Sm. & 3 & Huilunte & PMH, PA & $\mathrm{aH}, \mathrm{aT}$ & $2300-4000$ & LC, $\mathrm{Pa}_{\mathrm{a}}$ & G. Calatayud 4579 (AMAZ, CUZ, USM) \\
\hline Qrthaeg bullate N.R. Salinas \& Pedraza & 3 & Qxcserapuli & BMH & $\mathrm{aH}, \mathrm{aT}$ & $2425-2800$ & $\mathrm{Ca}, \mathrm{Pa}_{\mathrm{a}} \mathrm{LC}$ & L. Valenzuela et al., 2842 (CUZ, MO, USM). \\
\hline Orthaeg sernesiflerg N.R. Salinas \& Pedraza & 3 & & BM & $\mathrm{aH}, \mathrm{aT}$ & $2769-3500$ & $\mathrm{Ur}, \mathrm{LC}$ & I. Huamantupa et al. 3834 (CUZ) \\
\hline Qrthaea ferrerrzae A.C. Sm. & 3 & & BM & $\mathrm{aH}, \mathrm{aT}$ & $600-2200$ & $\mathrm{Ux}, \mathrm{LC}$ & I. Huamantupa et al. gn (CUZ) \\
\hline Qrthaed pinnatinervig Mansf. & 2 & Mulluntux & BM & $\mathrm{aH}, \mathrm{aT}$ & $3300-3400$ & $\mathrm{LC}, \mathrm{Pa}_{\mathrm{a}}$ & A. Weberkauer 6925 \\
\hline Pernettexa prestrata (Cax.) DC. & 3 & Macha macha, Puna manzana, Tüac & BMH & sat & $2100-4700$ & $\mathrm{An}, \mathrm{Ca}, \mathrm{LC}, \mathrm{Pa}, \mathrm{Qu}, \mathrm{Ur}$ & L. Valenzuela 9717 (CUZ, MO, USM) \\
\hline Psammisia amazonica Lutevn & 3 & Monte api & $\mathrm{AM}$ & $\mathrm{aH}, \mathrm{aT}$ & $300-700$ & Ux & P. Nux̃̃ \\
\hline Psammisia cearctata (Ruiz \& Pay.) A.C. Sm & 1 & Sarulille & BMH & $\mathrm{aH}, \mathrm{aT}$ & $1460-2500$ & LC & G. Calatayud et al., 3301 (CUZ, MO, USM). \\
\hline Psammisia ulkrichigna Hoerold & 1 & & $\mathrm{BM}, \mathrm{BA}$ & $\mathrm{aH}, \mathrm{aT}$ & $1800-2800$ & $\mathrm{~Pa}_{3}, \mathrm{LC}, \mathrm{Ur}$ & G. Calatayud et al., 2199 (AMAZ, CUZ) \\
\hline Satrrig pepurensiz (Benth. ex Meisn.) Ho f. ex Nied. & 2 & Monte api & $\mathrm{BA}$ & $\mathrm{aH}, \mathrm{aT}$ & $500-1500$ & $\mathrm{~Pa}_{\mathrm{a}} \mathrm{Qu}$ & F. Cornejo \& A. Balarezo 2751 (MO) \\
\hline Siphengandra ellintice Klotzsch & 3 & Huilluntuxy, Monte gapuli & BMH & $\mathrm{aT}$ & $2500-3800$ & $\mathrm{Ca}, \mathrm{LC}, \mathrm{Pa}, \mathrm{Ur}$ & I. Huamantupa 4440 (CUZ, MO) \\
\hline Spluzrespermum buesij A.C. Sm. & 1 & & BM & sath, sat & $1500-3000$ & $\mathrm{Ca}, \mathrm{LC}, \mathrm{Pa}, \mathrm{Ur}$ & D. N. Smith 4356 (MO) \\
\hline Splzzospermum buxifolium Poenp, \& Endh. & 1 & Salapa, Irxara & $\mathrm{BM}, \mathrm{BA}$ & saH, saT & $1600-2700$ & $\mathrm{Ca}, \mathrm{LC}, \mathrm{Pa}, \mathrm{Ur}$ & I. Huamantura 7351 (CUz, MO) \\
\hline Seburospermum cerdifolium Benth. & 1 & & $\mathrm{BM}, \mathrm{BA}$ & $\mathrm{saH}, \mathrm{saT}$ & $1500-3000$ & $\mathrm{Ca}, \mathrm{LC}, \mathrm{Pa}, \mathrm{Ur}$ & I. Huamantupa et al., 6431 (CUZ, MO) \\
\hline Thibaudia gerulata J. Rémy & 3 & & BM & $\mathrm{aH}, \mathrm{aT}$ & $2200-3100$ & $\mathrm{Ca}, \mathrm{LC}, \mathrm{Pa}, \mathrm{Ur}$ & I. Huamantupa 2340 (CUZ, MO, USM) \\
\hline Thibaudia diphrlla Dunal & 3 & & $\mathrm{BM}$ & $\mathrm{aH}, \mathrm{aT}$ & $2000-3000$ & $\mathrm{Ca}, \mathrm{LC}, \mathrm{Pa}_{4} \mathrm{U} r$ & F. L. Herrera 2004 (F, G, NY, S) \\
\hline Thibqudia dudlesi Lutern & 3 & & BM & aH, aT & $1500-2600$ & $\mathrm{Ca}, \mathrm{LC}, \mathrm{Pa}_{\mathrm{a}} \mathrm{Ur}$ & T. R. Dudley 10688 (NA, USM) \\
\hline Thibgudia floribunda Kunth & 2 & Huilunter & BM & $\mathrm{aH}, \mathrm{aT}$ & $2200-3000$ & $\mathrm{Ca}, \mathrm{Ur}, \mathrm{LC}$ & L. Valenzuela et al., 3852 (CUZ, MO) \\
\hline Thibqudia raubij A.C. Sm. & 2 & & BM & $\mathrm{aH}, \mathrm{aT}$ & $520-1300$ & $\mathrm{Ca}, \mathrm{LC}, \mathrm{Pa}, \mathrm{Ux}$ & D. C. Warchausen 800 (MO) \\
\hline Thibaudia resularis A.C. Sm. & 2 & Mulluntux & $\mathrm{BM}$ & $\mathrm{aH}, \mathrm{aT}$ & $2400-3000$ & $\mathrm{Ca}, \mathrm{LC}, \mathrm{Pa}, \mathrm{Ur}$ & J. West 8020 (MO) \\
\hline Vaccinium dererdens (G. Don) Sleumer & 1 & Tumana & $\mathrm{PA}$ & sath & $2900-3200$ & $\mathrm{Ca}, \mathrm{LC}$ & L. Valenzuela et al., 882 (CUZ, MO) \\
\hline Vaccinium floribundum Kunth & 3 & Tumana, Alke macha macha & BM, BMH & sath & $2400-3600$ & $\mathrm{Ca}, \mathrm{LC}, \mathrm{Pa}_{\mathrm{s}} \mathrm{Ur}_{\mathrm{r}}, \mathrm{Qu}$ & G. Calatayud 1664 (CUZ, MO, USM) \\
\hline
\end{tabular}

Los hábitos están representados por: $\mathrm{A}=$ árbol, arbusto terrestre $=\mathrm{a} \mathrm{T}$, arbusto hemiepífito $=\mathrm{aH}$, suarbusto terreste $=$ saT, subarbusto hemiepifito $=$ saH 


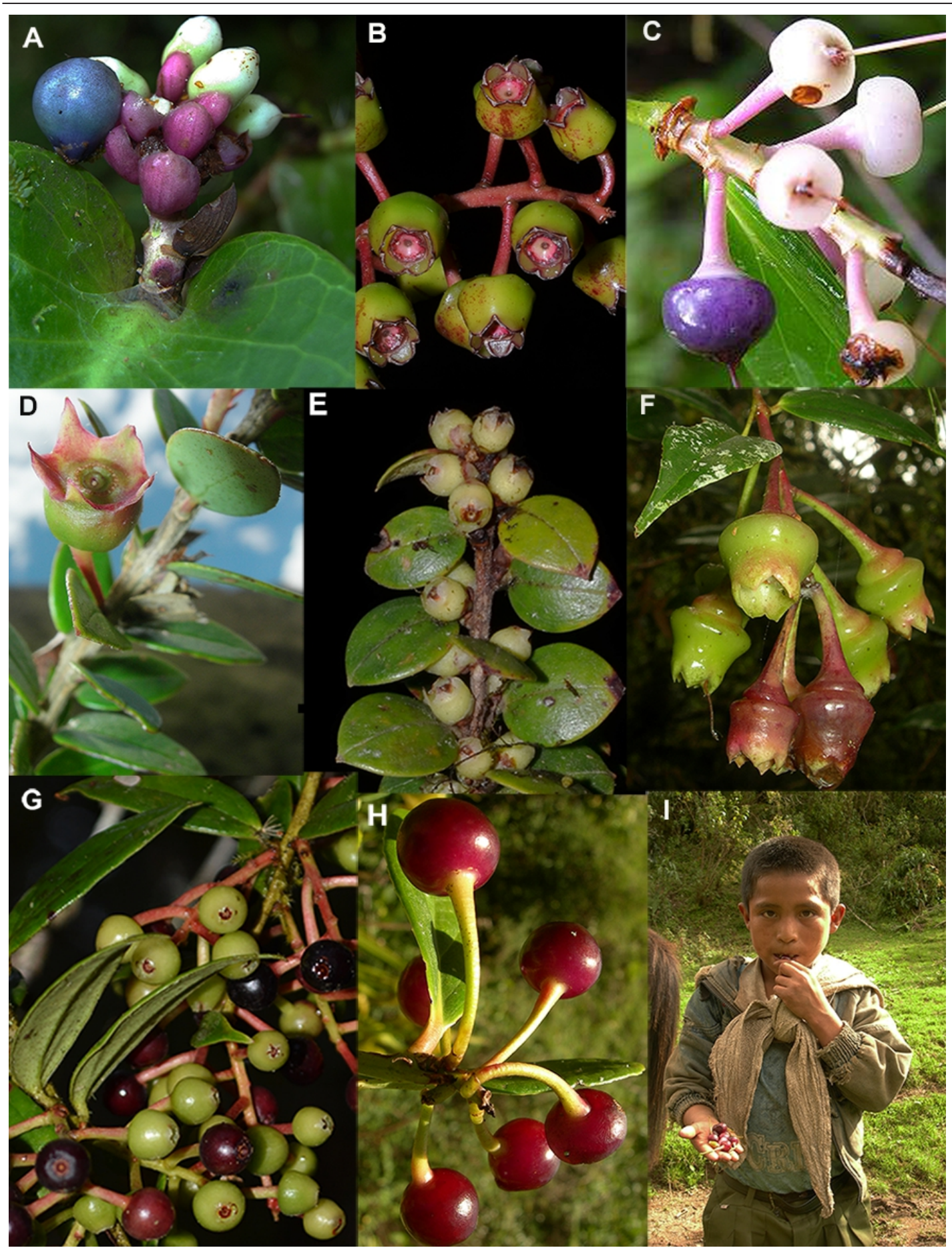

Figura 1. Ericáceas con frutos comestibles en la región Cusco. A. Cavendishia complectens, B. C. martii, C. C. nobilis (Foto B. Chambi), D. Demosthenesia mandonii, E. Disterigma ovatum, F. Orthaea carnosiflora, G. O. bullata, H. Macleania rupestris e I. Niño local consumiendo frutos de M.rupestris. (Fotografías I. Huamantupa). 

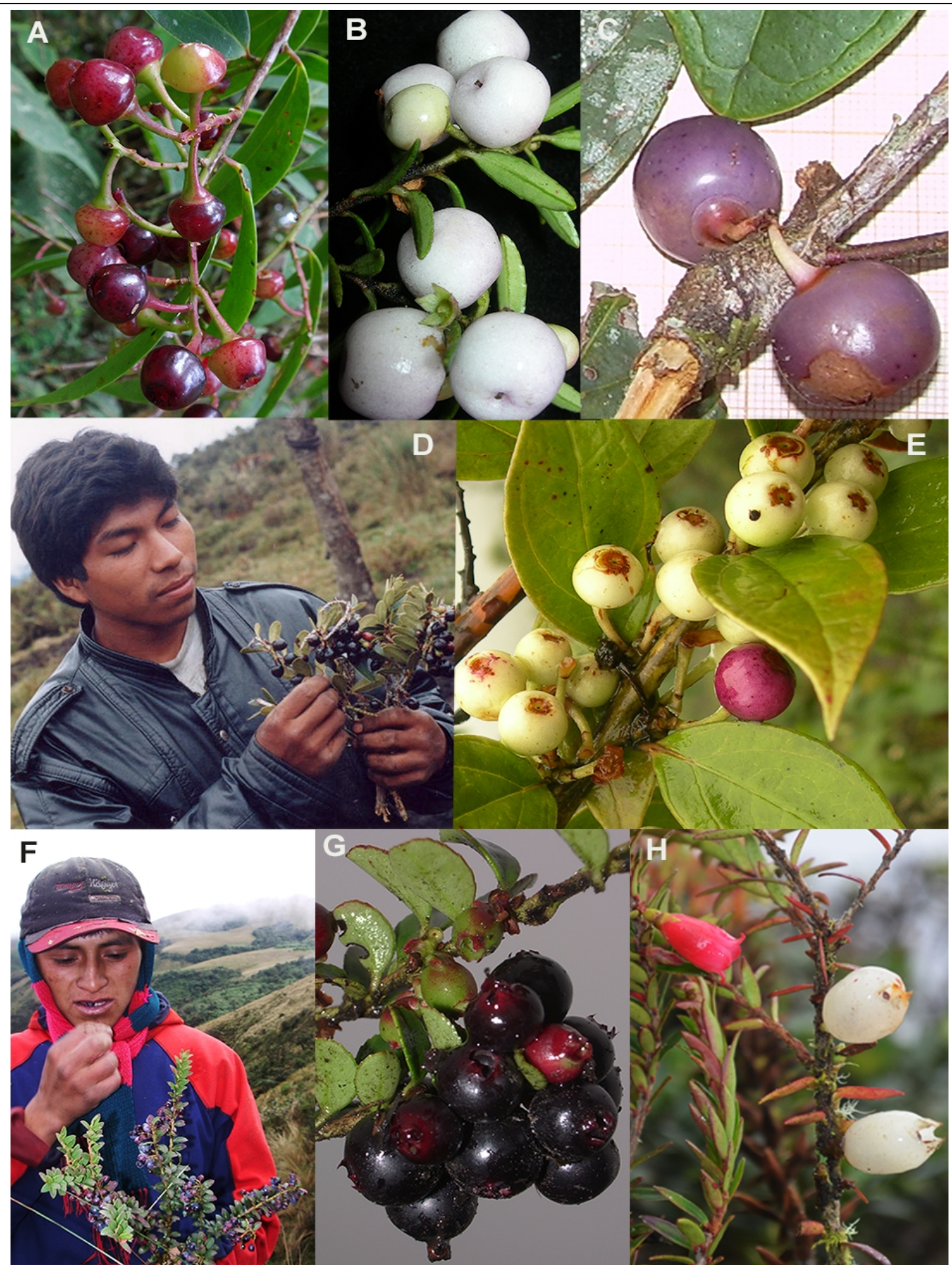

Figura 2. Ericáceas con frutos comestibles en la región Cusco. A. Orthaea ferreyrae, B. Pernettya prostrata, C. Psammisia amazonica (Foto P. Nuñez), D. Poblador consumiendo frutos de Siphonandra elliptica, E. Thibaudia crenulata, F. Poblador consumiendo frutos de Vaccinium dependens, G. Vaccinium floribundum y H. Disterigma empetrifolium. (Fotografías I. Huamantupa). 
De nuestras investigaciones las especies que como cultivados en la provincia de La tienen un alto potencial de aprovechamiento Convención en las partes altas de la localidad de para frutales y que puedan tener una aceptación Mesa pelada. Las especies Orthaea carnosiflora, $O$. en el mercado son 10 especies (Tabla 2), estas ferreyrae y Psammisia amazonica, corresponden destacan por tener un sabor dulce agradable, su aquellas que no tienen evidencias de cultivo, mesocarpio abundante y su abundante pero son frecuentemente consumidas por los producción en las ramas.

pobladores locales, estas son quizá las que

Se evidencio prácticas de manejo en pequeños cultivos locales de Demosthenesia En algunos mercados locales se evidencio la mandonii, Orthaea bullata, Siphonandra elliptica y venta de Vaccinium floribundum y Vaccinium floribundum en la provincia de esporádicamente el de Thibaudia crenulata y Paucartambo en las localidades de Challabamba Pernettya prostrata, de estas V.floribundum es la que y Pilco grande, las cuales muestran un éxito de tiene mayor demanda y son expendidas desarrollo y fructificación en pequeñas huertas frecuentemente en diversos mercados, de las casas aledañas a los lugares donde crecen incluidas algunas grandes como el mercado de de forma silvestre. Las especies Macleania Vinocanchon de San Jerónimo de la ciudad del rupestris yVaccinium foribundum fueron registrados Cusco (Figura 3).

Tabla 2. Especies de Ericaceae frutales con altos potenciales para su manejo en sistemas agroforestales.

\begin{tabular}{llcl}
\hline \multicolumn{1}{c}{ Especie } & Nombre vernáculo & $\begin{array}{c}\text { Fruto } \\
\text { diámetro } \\
\text { (cm) }\end{array}$ & \multicolumn{1}{c}{ Tipo manejo } \\
\hline \hline $\begin{array}{l}\text { Demosthenesia mandonii } \\
\text { Macleania rupestris }\end{array}$ & Mulluntuy & $1.5-2$ & Silvestre, cultivado \\
Orthaea bullata & Orcco capuli & $2-3$ & Silvestre, cultivado \\
Orthaea carnosiflora & & $1-2$ & Silvestre, cultivado \\
Orthaea ferreyrae & Macha macha & $2-3$ & Silvestre \\
Pernettya prostrata & Monte api & $2-3$ & Silvestre \\
Psammisia amazonica & Huilluntuy, Monte & $2-3$ & Silvestre, cultivado \\
Siphonandra elliptica & capuli & $2-3$ & Silvestre \\
Thibaudia crenulata & & $2-3$ & Silvestre, cultivado \\
Vaccinium floribundum & Tumana & $1-2$ & Silvestre, cultivado \\
\hline
\end{tabular}

Estado de conservación de Ericáceas con frutos comestibles

Se registró 13 especies con categorías de amenaza, de ellas 10 están consideradas en peligro crítico (CR), 1 en peligro (EN) y 1 con datos insuficientes (DD). Los géneros con más especies con categoría de amenaza es Demosthenesia con 7 especies, seguida de Thibauida con 3 especies (Tabla 3).
La mayor parte de estas especies con categorías de amenaza están presentes en las provincias de La Convención, seguidas en similar proporción en Calca, Paucartambo y Urubamba. 
Tabla 3. Especies de Ericaceae frutales con categorías de amenaza en la región Cusco.

\begin{tabular}{llllc}
\hline Especie & $\begin{array}{l}\text { Nombre } \\
\text { vernacular }\end{array}$ & Habito & Provincias & $\begin{array}{l}\text { Estatus } \\
\text { poblacional }\end{array}$ \\
\hline \hline Demosthenesia amicorum & Mulluntuy & Arbusto, hemiepífito & LC, Ur & CR \\
Demosthenesia buxifolia & Mulluntuy & Arbusto hemiepífito & LC & EN \\
Demosthenesia cordifolia & Mulluntuy & Arbusto & LC & CR \\
Demosthenesia dudleyi & Mulluntuy & Arbusto & LC & CR \\
Demosthenesia matsiguenka & & Arbusto hemiepífito & LC & CR \\
Demosthenesia vilcabambensis & Mulluntuy & Arbusto & LC, Ca & CR \\
Demosthenesia weberbaueri & Mulluntuy & Arbusto & LC & CR \\
Disterigma ulei Sleumer & & Hierba palustre & $\mathrm{Pa}$ & $\mathrm{DD}$ \\
Sphyrospermum buesii & & Hierba palustre hemiepífita & $\mathrm{Ca}, \mathrm{LC}, \mathrm{Pa}, \mathrm{Ur}$ & $\mathrm{EN}$ \\
Thibaudia diphylla & & Arbusto & $\mathrm{Ca}, \mathrm{LC}, \mathrm{Pa}, \mathrm{Ur}$ & $\mathrm{CR}$ \\
Thibaudia dudleyi & Arbusto & $\mathrm{Ca}, \mathrm{LC}, \mathrm{Pa}, \mathrm{Ur}$ & $\mathrm{CR}$ \\
Thibaudia rauhii & & Arbusto & $\mathrm{Ca}, \mathrm{LC}, \mathrm{Pa}, \mathrm{Ur}$ & $\mathrm{CR}$ \\
\hline
\end{tabular}

\section{Discusiones}

\section{Diversidad de Ericáceas frutales en la región Cusco}

La presencia de 51 especies de Ericáceas con los frutos consumidos representa una gran diversidad de plantas que tienen una tradición de consumo en seis provincias de la región Cusco, la cual está directamente relacionada a las costumbres provenientes desde épocas preincas e inca. Estos resultados contribuyen enormemente al incremento del conocimiento de especies frutales y a su vez estas ofrecen oportunidades para ser manejadas en un futuro próximo.

En cuanto a la riqueza de taxones (taxa) el presente estudio a nivel regional para el ámbito de Cusco difiere notablemente a los trabajos de Huamantupa (2010) en la que se registra 14 especies de Ericaceae con frutos y corolas de las flores que se consumen, donde destacan los géneros Demosthenesia, Orthaea, Psammisia y Vaccinium. En el mismo contexto a los registros realizados por Vargas (1994) quién menciona a 15 especies con los frutos consumidos, de ellas destaca a las especies Demosthenesia mandonii, D. spectabilis y Thibaudia crenulata conocidas vernacularmente como "mulluntuys" y Vaccinium floribundum como "tumana", haciendo énfasis que estás mayormente se encuentran en las provincias de La Convención y Paucartambo, estas fueron registradas en las mismas provincias y otras en la región Cusco en el presente estudio.

Por otro lado, Brack (1999) y Mostacero et al. (2009), mencionan para Perú un poco más de 20 especies con frutos comestibles de Ericaceae, en su mayoría destacan a especies del norte peruano, a los departamentos de Amazonas, Cajamarca y La Libertad, de ellas se corroboran varias en nuestro estudio sobre todo especies del género Cavendishia, Macleania, Satyria y Siphonandra.

A nivel de la región andina neotropical nuestro estudio reporta una mayor riqueza de especies con respecto a otros estudios como los de Abril (2010) quién identifica 11 especies de Ericáceas consumidas en el Altiplano Cundiboyacense de Colombia, de las cuales se 
comparte con las especies cusqueñas a: Cavendishia bracteata, C. pubescens, Gaultheria erecta, G. sclerophylla, Maclenia rupestris y Thibaudia floribunda. En el mismo sentido nuestro registro es considerablemente amplio a la lista mencionada por de LaTorre et al. (2008) quienes hacen una recopilación de las especies útiles de plantas para Ecuador, en la que Ericaceae está representada por 31 especies con frutos comestibles, conformadas por especies de bosque montano y Amazonia en mayor proporción, en estas destacan 17 especies en común con nuestro estudio: Cavendishia bracteata, C. nobilis, C. tarapotana, Disterigma acuminatum, D. alaternioides, D. humboldti, D. microphyllum, D. pernettyoides, Gaultheria glomerata, Maclania floribunda, M. rupestris, Pernettia prostrata, Psammisia ulbrichiana, Styria polyantha, Sphyrospermum buxifolium, Thibaudia floribunda yVaccinium floribundum.

Estos registros en común tanto para Colombia y Ecuador, evidencia la amplitud de su distribución geográfica asociados a los diferentes hábitats en común y a su amplitud de resiliencia ecológica que tienen estás plantas que sin duda son una ventaja frente a otros grupos. Esta amplitud de resiliencia en nuestro estudio está representada por tres gradientes marcadas siendo de los 2000 a 3500 msnm que comprende al bosque montano alto, bosque montano bajo, bosque de transición de pajonal y arbustal, seguida entre especies presentes entre los 1000 hasta los 2000 msnm, y en menor proporción especies amazónicas, de zonas altoandinas por encima de los $3500 \mathrm{msnm}$. Leiva et al. (2013) identifica 7 especies de Ericaceae que frecuentemente son consumidas en los andes centrales neotropicales en los ámbitos de Bolivia, Ecuador y Perú, trabajo con el que se menciona en común a nuestro estudio a las especies Disterigma empetrifolium,
D. humboldtii, Gaultheria erecta y Vaccinium foribundum, a las cuales hace referencia que tienen un alto potencial vitamínico.

A nivel de género se distingue a Demosthenesia la que tiene mayor diversidad de especies representadas por 10 especies, esto hace denotar que estos frutales son de preferencia por el recurso que ofrece los frutos que en todas ellas son bayas suculentas de color morado a negruzcas, con sabor dulce y de pulpa suculenta. Contrariamente hacia latitudes menores al norte peruano Demosthenesia no esta representada por especie alguna, al igual que Siphonandra, ambas consideradas endémicas de los andes peruanos y bolivianos (Luteyn \& Ortiz, 2008). El otro género rico en especies es Disterigma con 8 especies, que en su mayoría corresponde a especies sub arbustivas que gozan de una regular y poca frecuencia de consumos dado que los frutos son pequeños llegando hasta $1.5 \mathrm{~cm}$ de diámetro en el mejor de los casos, habitan de pajonales y bosques montano húmedos transicionales y Cavendishia 6 especies representada por arbustos terrestres y hemiepífitos en los bosques montanos principalmente. Ambos géneros tienen una amplia distribución en los andes neotropicales y de forma similar son consumidas sus frutos.

De nuestros registros las especies que tienen mayor frecuencia de consumo para la región Cusco son 12, de estas destacan en la zona altoandina y cercanas a valles interandinos como en la parte alta del Parque Nacional del Manú, Lacco Yavero en Paucartambo, Amparaes y Lares en Calca, zonas altas de Urubamba, en las zonas de Marcapata en Quispicanchis y en los ámbitos de Punkuyoc, Carrizales, Huyro, Mesa Pelada, Vilcabamba, en la provincia de la Convención, con las especies: Vaccinium floribundum, Siphonandra 
elliptica, Orthaea bullata y Pernettya prostrata. Registros interesantes en cuanto a su frecuencia de consumo alto son: Macleania rupestris que hasta años anteriores sólo se conocían para el norte y centro peruano, pero durante nuestro estudio se pudo corroborar que esta especie al igual que en el norte de los andes neotropicales goza de amplia frecuencia de consumo dado que sus frutos superan en algunos casos los $3 \mathrm{~cm}$ de diámetro, de la misma forma sucede con el hallazgo de Psammisia amazonica, que ahora hemos registrado en el bajo Urubamba de la provincia de la Convención donde las comunidades Matshiguenkas las consumen frecuentemente, esta especie hasta antes del presente estuvo referenciada para el centro y norte amazónico peruano, esta especie goza de amplia aceptación de consumo en las comunidades amazónicas, donde los frutos de color morado lila son de apreciable suculencia y sabor dulce.

A pesar de haberse registrado 51 especies en el presente estudio, creemos que aún existen varias especies no inventariadas en otros lugares de la región Cusco que de seguro incrementaran esta lista de taxones. Una de las formas de consumo también son la ingesta de corolas principalmente en los géneros Psammisia y Orthaea, estos registros en el presente estudio no se tomaron en cuenta.

\section{Potencialidades de Ericáceas frutales en la región Cusco}

En el ámbito de trabajo existen las potencialidades intangibles, en mayor medida. La significación que tengan para cada una de las zonas, en términos de gestión y manejo, dependerá del nivel de uso o aprovechamiento actual que se haya logrado de las mismas.
Los registros de manejo y consumo de Ericaceae en la región andina del Perú, se remontan hasta los antiguos peruanos, datos registrados en algunas crónicas, enfatizan que hasta antes de la llegada de los europeos, se tenía un consumo frecuente de especies frutales de Ericaceae, conocidos con los nombres de huilintuy, tumana, mortiño entre otros, que eran consumidas directamente, utilizadas para preparar mazamorras en festividades tradicionales, como tintes colorantes y otros (Cieza de León, 1962; Estrella, 1986), estas especies correspondan a Vaccinium floribundum y posiblemente a Pernettia prostrata, los cuales hasta ahora en algunos mercados locales como el de la ciudad de Paucartambo se siguen utilizando de diversas formas, haciendo referencia a que estas costumbres y conocimientos provienen desde sus antepasados incas.

Entre todas las 51 especies, consideramos a 10 como las de mayor potencial de cultivo como frutal para consumo directo por los registros y evidencias de cultivos locales siendo: Demosthenesia mandonii, Macleania rupestris, Orthaea bullata, Pernettya prostrata, Siphonandra elliptica, Thibaudia crenulata y Vaccinium floribundum las que vienen siendo manejadas localmente pero que estas iniciativas sin duda muestran que en la región del Cusco existen lugares con alto potencial para un manejo mayor.

De acuerdo con nuestras observaciones y análisis creemos que los arbustos Orthaea carnosiflora, O. ferreyrae y Psammisia amazonica deben de ser investigadas para su aprovechamiento, puesto que son de las especies que tienen los mayores frutos dentro de las Ericaceae, con agradable sabor dulce y abundante mesocarpio suculento. 
En todas las especies los frutos son cosechados directamente de las ramas, cuando están maduros o relativamente maduros como en el género Orthaea. Frecuentemente son consumidas directamente de la cosecha o pueden ser llevadas para el consumo en los hogares - sólo algunas especies son cosechadas para la venta en mercados locales como: Macleania rupestris, Siphonandra elliptica, Thibaudia crenulata y Vaccinium floribundum (Figura 3), de ellas esta última destaca por su incremento de ventas en los diferentes mercados locales de Cusco, donde los vendedores las promocionan como los "arandanos de los andes".V.floribundum es una de las pocas especies que en los últimos años ha tenido mayor demanda en los mercados locales de la región andina peruana (Mostacero, 2017) y en otros países como en Colombia y Ecuador conocido como mortiño (Sanjinez et al., 2006).

Cabe resaltar que el género Vaccinium aparentemente tiene ventajas para su cultivo ya que es el género que tiene más especies cultivadas como sucede con Vaccinium corymbosum, V. ashei, y V. angustifolium, en Norteamérica como manifiesta Gaviria et al. (2009).

Otra especie importante es Macleania rupestris presente en algunas provincias del Cusco, tiene un enorme potencial para su aprovechamiento, dado que en otros países como en Ecuador con nombre walicon, los frutos son expendidos en los mercados locales de Quito y Otavalo, con alta demanda de consumo (observación personal del 1er autor), esta misma en Colombia conocida como uva camarona y uva de monte viene siendo investigada en términos de producción y aprovechamiento, ya que goza de excelentes cualidades como de agradable sabor una pulpa sustanciosa, que además vienen transformándolas en derivados como dulces, mermeladas y otros en la culinaria (Corzo, 2014).

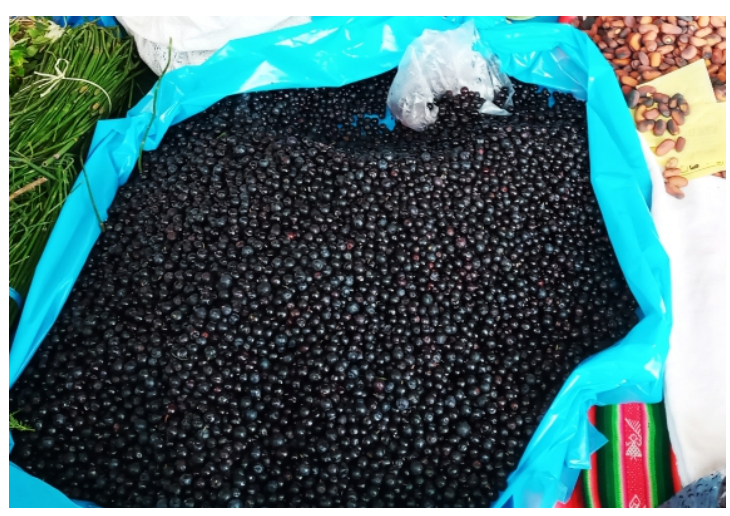

Figura 3. Vaccinium floribundum "tumana" expendido en el mercado de VinocanchonCusco.

Actualmente existe una tendencia al incremento de cultivos locales en las chacras y huertas por la relativa facilidad de trasplante de plántulas juveniles extraídas de sus hábitats en las zonas adyacentes a donde crecen, este es el caso de especies de los géneros Demosthenesia, Disterigma, Macleania, Orthaea y Siphonandra.

Sin duda, uno de los potenciales mayores de las Ericaceae hoy en día son los referidos a los atributos nutraceúticos, por la presencia de diversos metabolitos primarios y secundarios. Uno de estos es el caso de la ya conocida Vaccinium floribundum y otras del mismo género que en los frutos contienen diversos azúcares, minerales, antioxidantes, vitaminas del complejo B, C y minerales como potasio, calcio, fósforo, proantocianidinas, antocianidinas, flavonoides, que se utilizan como colorantes alimentarios, las cuales además se aplican para curar y aliviar afecciones y enfermedades como el reumatismo, fiebres, cólicos y otros (Morales, 2011; CESA, 1993; Abreu et al., 2008; Mostacero et al., 2017). Estas propiedades mencionadas han hecho además que a varias especies de Ericaceae se las considere como medicinales como la 
aplicación contra la diabetes al ser consumidas crudas y maduras, las cuales ayudan a compensar los niveles de azúcar en la sangre, así como también otros problemas estomacales y cardiacos gracias a sus propiedades antioxidantes (Aguilar, 2009; Gaviria et al., 2007).

En la especie Macleania rupestris también se ha reportado la presencia de altas proporciones de vitamina $\mathrm{C}$ en los frutos, en las hojas presentan taninos, flavonoides, cumarinas y esteroides (García-Barriga, 1975; Plazas, 2013), por lo cual actualmente se viene incrementando su cultivo, principalmente en Colombia y Ecuador (Corzo, 2014). Al igual que estas especies conocidas algunas otras se vienen estudiando por estos potenciales nutraceúticos. Plazas (2015) en su estudio fitoquímico devela que para las especies Cavendishia bracteata, Gaultheria erecta y Thibaudia floribunda poseen componentes antioxidantes, especies que se registraron en nuestro estudio.

Los compuestos antioxidantes al parecer son frecuentes en todos los géneros de Ericaceae como se expresa en el estudio de Dastmalchi et al. (2011) se evidencia de manera particular en cada especie los compuestos antioxidantes destacando con alta proporción de estas en las especies que son consumidas sus frutos: Cavendishia grandiflora y Anthopterus wardii a diferencia de Macleania coccoloboides, Sphyrospermum buxifolium y Sphyrospermum cordifolium. Cabe resaltar algunas especies presentan ciertos componentes toxicas como en Cavendishia bracteata (Plaza, 2015) esto se evidencia en la frecuencia de consumo de varias especies cusqueñas como sucede en 21 especies que solamente están representadas por una escasa frecuencia de consumo (1), en ellas destacan los géneros Cavendishia, Gaultheria y
Disterigma a los cuales en varias localidades se las conoce como macha macha (traducido al castellano emborrachadoras). Aparentemente las especies que tienen los frutos coloridos de lila, violeta, azul y negruzcos son las que poseen mayor frecuencia de consumo, los cuales estarían asociados a presentar mayores compuestos nutraceúticos, entre ellos metabolitos secundarios como fenoles, flavonoides, antocianinas y otros como lo manifiestan algunos estudios como los de Aguilar, (2009), Gaviria et al. (2012) y Morales (2011).

Otro potencial que poco en la región del Cusco y el Perú es poco considerado el aprovechamiento como ornamental, en la que las Ericaceae tienen muchas ventajas frente a otros grupos, por el hábito arbustivo que tienen una aparente facilidad de manejo, por las vistosas flores que presentan como en los géneros Cavendishia, Psammisia, Thibaudia, Macleani, Demosthenesia y Siphonandra que acompañadas de los frutos que se consumen que a su vez pueda servir a la fauna nativa como a varios polinizadores y dispersores como las aves andinas.

Lo relativo al nuevo enfoque de potencialidades, para las Ericáceas con sus diversos usos como en la alimentación y el aprovechamiento del fruto, es la falta de disponibilidad de información cuantitativa de referencia sobre distintos aspectos para la gestión y manejo de las especies reportadas. La información en cuanto a las potencialidades de manejo en la región del Cusco y el Perú de Ericáceas en general aún se conoce muy poco, pero la registrada en la presente que proviene de un conocimiento rico de los habitantes locales, nos permite sentar las bases para el aprovechamiento de sus potencialidades. 


\section{Conservación y amenazas de} Ericáceas frutales en la región Cusco

Las especies consideradas con categorías de amenaza corresponden representan al 20\% del total registrado en el presente estudio, estas están relacionadas principalmente aquellas que tienen una distribución geográfica restringida, como en el caso de las especies del género Demosthenesia en la cual son 6 las endémicas de la cordillera del Vilcabamba. Sin duda las especies con categorías de amenaza hacen denotar que a pesar de que las Ericaceae son poco conocidas y gozan de escasas experiencias de aprovechamiento, están vinculadas al peligro de desaparecer dado que tienen una distribución en pequeñas áreas como son los casos de $D$. cordifolia, D. dudlei, D. matshiguenka y D. vilcabambensis, que sólo se conocen de las colectas tipo en la provincia de La Convención.

Indudablemente las especies de Ericaceae como una de las familias de plantas de mayor diversificación en los andes del Neotrópico (Kron et al., 2002) está asociada a sus hábitats particulares que demandan condiciones abióticas especiales que varían rápidamente en una gradiente altitudinal, tales como la disponibilidad de nutrientes en el suelo, regímenes de precipitación, humedad, temperatura, las horas luz, disponibilidad de agua en el suelo y a su vez estos relacionados a sus dispersores y polinizadores específicos que en gran parte están representadas como polinizadores los colibríes.

Las especies registradas como frutales en la región del Cusco, además de tener altos potenciales son también sensibles a cambios en sus hábitats como el cambio de uso de la tierra por la ganadería y agricultura, la deforestación y pérdida de hábitats. Por ello consideramos que se debe hacer estudios más profundos y categorizarlas a nivel regional. Algunos ejemplos de especies con alta frecuencia de consumo y con categorías de amenaza son Thibaudia dudlei, en peligro crítico (CR) sólo conocida de la descripción tipo y consumida en las zonas altas de las localidades de Puncuyoy y Vilcabamba en la provincia de la Convención, en los casos de Orthaea carnosiflora y O.ferreyrae deben de ser consideradas como en peligro (EN), que si bien es cierto se comparten con los departamentos de Huánuco y Pasco, en Cusco solo se han registrado en pocas localidades donde están amenazadas sus poblaciones por la expansión agrícola.

\section{Conclusiones}

El estudio registró 51 especies de Ericáceas con frutos consumidos por los pobladores locales, distribuidas en 14 géneros. Siendo los géneros más diversos Demosthenesia con 10 especies, Disterigma (8), Cavendishia y Thibaudia con 6, este registro de alta diversidad es considerablemente mayor a los estudios previos, tanto a nivel local y en los otros países donde también las Ericaceae son diversas. De las 51 especies, 12 son las especies que gozan de mayor frecuencia de consumo; y 10 especies presentan con alto potencial para ser manejados en cultivos locales, como nutraceuticos y otros, de ellas actualmente son expendidas en algunos mercados locales: Vaccinium floribundum y esporádicamente Thibaudia crenulata y Pernettya prostrata, una limitante para la alimentación y el aprovechamiento de estos frutos es que existen escasos estudios enfocados a investigar las especies frutales silvestres y sus potenciales. En lo referente al estado de conservación hemos determinado 13 especies con categorías de amenaza, de ellas 10 están consideradas en 
en peligro crítico (CR), siendo Demosthenesia el género con más especies categorizadas, sin embargo, se plantean que algunas de ellas deben de re categorizarse en la región del Cusco como sucede Orthaea carnosiflora y O.ferreyrae, asociadas además a su limitada distribución geográfica.

Estos resultados evidencian la existencia de una enorme riqueza de especies de la familia Ericaceae con los frutos consumidos en la región del Cusco, algunas de estas actualmente manejadas y aprovechadas por las poblaciones locales, los que son considerados como recursos intangibles con valor indefinido. Este enorme potencial está representado por especies con aptitudes agronómicas, con potencial nutraceútico y medicinal, como ornamentales y otros. En este sentido a partir de los grandes atributos y beneficios con el consumo de los frutos de Ericaceae, alentamos a que se hagan estudios para un aprovechamiento adecuado y que a su vez sean parte de alternativas futuras de desarrollo, y que mejoren la calidad de vida de las poblaciones locales.

\section{Agradecimientos}

El primer autor dedica la presente obra y a la vez agradece a su querida Madre Claudia Chuquimaco, por haber sido la fuente de inspiración para estudiar las Ericáceas, gracias a que cuando niño en las diversas visitas a los abuelos que moraban en las localidades de Challabamba, Cajahuana y los pajonales de Accanacu adyacente al Parque Nacional del Manu, dentro de la provincia de PaucartamboCusco, ella con su conocimiento enseñó a distinguir y consumir estos frutales silvestres, conocidos localmente como mulluntuys, tumanas y macha machas.

Agradecemos a los pobladores de las diversas comunidades de las provincias de Cusco que nos brindaron su apoyo con la información etnobotánica de las Ericaceae, que, sin duda sin ese aporte del valioso conocimiento ecológico tradicional, no hubiera sido posible documentar las especies frutales que a su vez son legados de conocimientos ancestrales.

Agradecemos al herbario Vargas (CUZ), como institución científica perteneciente a la Universidad Nacional de San Antonio Abad del Cusco, que gracias a su acervo fue posible la sistematización de las Ericaceae a lo largo de varios años, producto de colecciones realizada por diversos botánicos. Al Dr. James Luteyn por su colaboración y apoyo en el conocimiento taxonómico de las Ericaceae durante su visita al Cusco en los años 2002 y 2003. Al New York Botanical Garden y al Missouri Botanical Garden, por habernos acogido durante nuestra visita a sus acervos donde pudimos incrementar nuestros conocimientos sobre las Ericaceae.

\section{Literatura Citada}

Abreu, A., Cuellar, A. \& Prieto. S. (2008). "Fitoquímica del género Vaccinium (Ericaceae)". Revista Cubana de Plantas Medicinales (13).

Abril, R. D. L. (2010). Las ericáceas con frutos comestibles del Altiplano Cundiboyacense. Tesis de Grado. Pontificia Universidad Javeriana. Bogotá, Colombia.

Aguilar, Z. (2009). Guía de plantas útiles de los páramos de Zuleta, Ecuador. EcoCiencia, Proyecto Páramo Andino. Programa de Apoyo a la Gestión Descentralizada de los Recursos Naturales en lasTres Provincias del Norte del Ecuador, pág. 99.

Aragón, R. J. I. \& Chuspe Z, M. E. (2018). Ecología geográfica del Cusco. Gobierno Regional del Cusco. Pp. 87.

Brack A. (1999). Plantas Útiles del Perú. Diccionario Enciclopédico. CBC. Cusco

Brako, L. \& Zarucchi. J. (1993). Catalogue of the Flowering Plants and Gymnosperms of Peru. Monographs in Systematic Botany of the Missouri Botanical Garden 45: 414-425. 
CESA. (1993). Usos tradicionales de las especies forestales nativas en el Ecuador, tomo 3. Central Ecuatoriana de Servicios Agrícolas, Quito - Ecuador, primera edición, pág. 258.

Cieza De León, P. (1962). Crónica del Perú. Espasa Calpe, Madrid España, tercera edición, pág. 249.

Corzo, B. D. C. (2014). Estudio del comportamiento poscosecha de Macleania rupestris (Kunth), en diferentes tipos de envases y condiciones de temperatura. Revista Iberoamericana de Tecnología Postcosecha, 15(1),77-82.

De la Torre, L., Alarcón, D., Peter-Kvist, L. \& Salazar, L. J. (2008). Usos medicinales de las plantas. En: L. De la Torre, H. Navarrete, P. Muriel, M. J. Macía, H. Balslev (eds.), Enciclopedia de las Plantas Útiles del Ecuador (pp. 105-114.). Herbario QCA7 \& Herbario AAU. Quito \& Aarhus.

Estrella, E. (1986). El pan de América: etnohistoria de los alimentos aborígenes en el Ecuador, tomo 29. Publicaciones del C.S.I.C. conmemorativas del V centenario del descubrimiento de América. Consejo Superior de Investigaciones Científicas, tercera edición

Garcia-Barriga, H. (1975). Flora medicinal de Colombia. Primera Edición. Imprenta Nacional.

Gaviria, C., J. D. Hernández., M. Lobo, C. I. Medina \& Rojano. B. A. (2012). Cambios en la actividad antioxidante en frutos de mortiño (Vaccinium meridionale sw.) durante el desarrollo y maduración. Revista de la facultad de agronomía Medellin, 65: 6487-6495.

Gonzales-Vigil, J. et al. (2003). Mapa de las Potencialidades del Perú. Una Primera Aproximación a Nivel Provincial. Programa de las Naciones Unidas para el Desarrollo-PNUD.Lima, Perú.

Huamantupa, C. I. (2008). Una nueva especie de Demosthenesia (Ericaceae) del Cusco, Perú. Revista Peruana de Biología 15(2): 79-81.

Huamantupa, C. I. (2010). Avances de la Sinopsis Taxonómica y Etnobotánica de la familia Ericaceae en el departamento Cusco. Acta Biol. Herreriana 1: 25 34.

Kron, K. A., Powell, E. A. \& Luteyn. J. L. (2002). Phylogenetic relationships within the blueberry tribe (Vaccinieae, Ericaceae) based on sequence data from matk and nuclear ribosomal its regions, with comments on the placement of Satyria. Amer. J. Bot., 89: 327-336.
Leiva, S., Zapata, M., Gayoso, G. \& Chang, L. (2013). Frutas silvestres con potencial vitamínico de los Andes Centrales de América. Arnaldoa 20(2): 315-358.

León, B. (2006). Ericáceas endémicas del Perú. En: Ed. Blanca León et al. El libro rojo de las plantas endémicas del Perú. Rev. peru. biol. Número e s p e c i a l $13(2): 285 \mathrm{~s}-293 \mathrm{~s}$. <http://sisbib.unmsm.edu.pe/BVRevistas/biolog ia/v13n2/pdf/a52.pdf $>$ (Acceso: 12/05/08).

León, B., Pitman, N. \& Roque. J. (2007). Introducción a las plantas endémicas del Perú. Rev. Perú. Biol. 13(2): 9s-13s.

Luteyn, J. L. (1983). Cavendishia. Flora Neotrópica. Monography. $\mathrm{N}^{\circ}$ 35: Pag. 1-290.

Luteyn, J. L. (1995). Ericaceae. New York: Organization for Flora Neotropica. New York: New York Botanical Garden.

Luteyn, J. L. (2002). Key to the species of Ericaceae of Bolivia, including two new species. Sida 20(1): 1-20.

Luteyn, J. L. (2004). Ericaceae. Pp. 140-143. En: N. Smith, S. A. Mori, A. Henderson, D. W. Stevenson \& S. V. Heald. Flowering plants of the neotropics. The NewYork Botanical Garden, NuevaYork.

Luteyn, J. L. \& Ortiz, E. (2008). Revision of Siphonandra (Ericaceae: Vaccinieae), a genus endemic to Peru and Bolivia. Journal of the Botanical Research Institute of Texas, 2(1), 249-261. Retrieved F e b r u a r y 2, $2021, \quad$ f r o m http://www.jstor.org/stable/41971623.

Luteyn, J. L. \& Pedraza-Peñalosa, P. (2018) (online). Neotropical blueberries: The plant family Ericaceae. The New York Botanical Garden. <www.nybg.org/bsci/res/lut2/> (Acceso: 12/05/18).

Moraes, M. R., Øllgaard, B., Kvist, L.P., Borchsenius, F. \& Balslev, H. (2006). Botánica económica de los andes centrales. Universidad Mayor de San Andrés. La Paz.

Morales, A. (2011). Frutoterapia, nutrición y salud Plus Vitae. EDAF del Plata, Madrid-España, primera edición, pág. 212. Padulosi S, Hoeschle-Zeledon I. ¿A que denominamos especies subutilizadas? LEISA.

Mostacero, J., Mejía, F. \& Gamarra, O. (2009). Fanerógamas del Perú: Taxonomía, utilidad y ecogeografía. CONCYTEC.Trujillo-Perú.

Mostacero, J., Rázuri, T. \& Gil, A. (2017). Fitogeografía y morfología de los Vaccinium (Ericaceae) “arándanos nativos" del Perú. Universidad Nacional deTrujillo. 
Padulosi, S. \& Hoeschle-Zeledon, I. (2004). ¿A que denominamos especies subutilizadas? LEISA revista de agroecológia; Junio: 6-8.

Pedraza-Peñalosa, P. (2010). Insensitive blueberries: a total evidence analysis of Disterigma s.l. (Ericaceae) exploring transformation costs.

Pedraza-Peñalosa, P. (2015). New blueberry and mortiño relatives (Ericaceae) from northwestern Colombia. PhytoKeys 49:33-58. doi: $10.3897 /$ phytokeys.49.8383

Pedraza-Peñalosa, P., Betancur, J. \& Franco, P. (2004). Chisacá, un recorrido por los páramos andinos. Segunda Edición. Universidad Nacional de Colombia. Bogotá., Colombia.

Plazas, E. A. (2013). Análisis fitoquímicos de tres especies vegetales para la apropiación del recurso florístico en la región Capital. Informe técnico inédito. Jardín Botánico de Bogotá.

Plazas, G. E. (2015). Tamizaje fitoquímico preliminar, evaluación de la actividad antioxidante in vitro y toxicidad de seis especies de Ericaeas colombianas. Revista de plantas medicinales cubana.

Sanjinés, A., Øllgaard, B. \& Balslev, H. (2006). Frutos comestibles. Botánica económica de los Andes Centrales. Universidad Mayor de San Andrés, La Paz.

Smith, N., Mori, S., Henderson, A., Stevenson, D. \& Heald. S. (2004). "Flowering plants of the Neotropics”. Princeton University Press, Princeton (United States),

Thiers, B. (2019). Index Herbariorum: A Global Directory of Public Herbaria and Associated Staff. New York Botanical Garden's Virtual Herbarium. http://sweetgum.nybg.org/ih/.

Vargas, C. C. (1994). Flora del Sur del Perú, Catalogo sistemático del Herbario Vargas (CUZ). Universidad Nacional de San Antonio del Cusco. Pgs. 197-2000. 